\title{
Uniqueness of the thermodynamic limit for driven disordered elastic interfaces
}

\author{
A. B. Kolton, ${ }^{1}$ S. Bustingorry, ${ }^{1}$ E. E. Ferrero, ${ }^{1, *}$ and A. Rosso ${ }^{2}$ \\ ${ }^{1}$ CONICET, Centro Atómico Bariloche, 8400 San Carlos de Bariloche, Río Negro, Argentina \\ ${ }^{2}$ LPTMS, Université Paris-Sud, CNRS (UMR 8626), 91405 Orsay Cedex, France
}

(Dated: January 12, 2018)

\begin{abstract}
We study the finite size fluctuations at the depinning transition for a one-dimensional elastic interface of size $L$ displacing in a disordered medium of transverse size $M=k L^{\zeta}$ with periodic boundary conditions, where $\zeta$ is the depinning roughness exponent and $k$ is a finite aspect ratio parameter. We focus on the crossover from the infinitely narrow $(k \rightarrow 0)$ to the infinitely wide $(k \rightarrow \infty)$ medium. We find that at the thermodynamic limit both the value of the critical force and the precise behavior of the velocity-force characteristics are unique and $k$-independent. We also show that the finite size fluctuations of the critical force (bias and variance) as well as the global width of the interface cross over from a power-law to a logarithm as a function of $k$. Our results are relevant for understanding anisotropic size-effects in force-driven and velocity-driven interfaces.
\end{abstract}

PACS numbers: 74.25.Qt, 64.60.Ht, 75.60.Ch, 05.70.Ln

\section{INTRODUCTION}

Understanding anisotropic finite-size effects in driven condensed matter systems is important not only for the custom numerical simulation analysis and modeling, but also to interpret an increasing amount of experiments performed on relatively small samples with specially devised geometries, where one of the system dimensions can be even comparable to a typical static or dynamical correlation length. The steady-state dynamics of directed elastic interfaces in random media, experimentally realized in driven ferromagnetic [1 5] and ferroelectric [6 10] domain walls, contact lines in wetting [11, 12] and fractures [13, 14], is a non-trivial relevant example were this kind of phenomenology arises. The study of driven domain wall motion in ferromagnetic micro-tracks for instance [15], relevant for memory-device applications or metallic ferromagnet spintronics [16], motivates the study of their motion in "wide" samples, i.e. much wider than the interface global width. Moreover, at the integration scale for modern nano-devices, these tracks can also become thin enough to be comparable to the typical size of the thermal nuclei controlling creep motion, thus yielding an experimentally observable dynamical dimensional crossover [17]. On the other extreme, periodic systems such as planar vortex lattices, charge density waves, or experimental realizations of elastic chains in random media, motivate, through an appropriate mapping, the study of the motion of large interfaces in periodically repeated "narrow" media, i.e. much narrower than the interface width 18 20].

Minimal models, such as the paradigmatic quenchedEdwards-Wilkinson equation (QEW) and their close quenched disorder variants [21], were shown to successfully capture experimentally observed universal dynam-

*Present address: LiPhy, Université Joseph Fourier, UMR 5588, 38041 Grenoble Cedex, France ics such as creep 1] and depinning [12, 22] phenomena. In spite of this, fundamental questions such as the possible thermodynamic limits of these models, when supplemented with the usual periodic boundary conditions, are not completely understood. Roughly speaking, the steady-state motion of extended elastic interfaces is expected to be very different in very narrow than in very wide samples because they actually sense rather different pinning force fluctuations from the same microscopic model. The thermodynamic limit in between these two extremes (i.e., not infinitely narrow nor wide samples), hence, looks rather ambiguous 23]: it is unclear whether it leads to a unique solution or to a family of solutions parametrized by some properly defined aspect-ratio parameter.

Let us consider a driven QEW one-dimensional interface in a disordered sample of dimensions $L \times M$, with periodic boundary conditions, as schematically shown in Fig. 1(a). For each sample, at zero temperature, a critical force $f_{c}^{s}$ separates a pinned phase from a sliding phase characterized by a finite velocity $v^{s}$. In finite systems, both $f_{c}^{s}$ and $v^{s}$ fluctuate from sample to sample and their averages over all disorder realizations, namely $\left\langle f_{c}^{s}\right\rangle$ and $\left\langle v^{s}\right\rangle$, depend both on microscopic details of the model (microscopic disorder distribution, lattice discretization, etc.) and the specific geometry of the sample (boundary conditions, transverse size, etc.). In Ref. [19] it was shown that if we choose $M=k L^{\zeta}$, with $\zeta$ the depinning roughness exponent, and a Gaussian microscopic disorder, the critical force distribution crosses over from a Gaussian (for $k \rightarrow 0$ ) to a Gumbel distribution (for $k \rightarrow \infty$ ) in the large $L$ limit. One can show that the "infinitely narrow" $k \rightarrow 0$ limit corresponds to the so-called random periodic (RP) depinning universality class, while the "infinitely wide" $k \rightarrow \infty$ limit corresponds to a dimensional crossover towards the zero dimensional case describing a single particle in an effective one-dimensional potential. In the former case, periodic effects arise when $M$ turns out to be comparable to the interface width, and becomes more and more impor- 
tant as $k$ decreases further and the interface winds more around the cylinder with perimeter $M$, as schematically shown in Fig. 1(b). In the latter case instead, as schematically shown in Fig. 1(c), periodicity effects are absolutely negligible but the roughness turns anomalous due to extreme value statistics effects [24]. In fact, for fixed $L$ and Gaussian disorder, $\left\langle f_{c}^{s}\right\rangle \rightarrow \infty$ as $M \rightarrow \infty$; so that, at zero temperature, a finite velocity is only possible in a nonsteady state 25]. The so-called random manifold (RM) regime, which exists between these two extreme limits for any finite $k$, was shown to display a finite critical force $f_{c}$ in the thermodynamic $\operatorname{limit}\left(f_{c}=\lim _{L \rightarrow \infty}\left\langle f_{c}^{s}\right\rangle\right)$, with sample to sample fluctuations vanishing as [19, 26, 27] $\left\langle\left[f_{c}^{s}-\left\langle f_{c}^{s}\right\rangle\right]^{2}\right\rangle \sim L^{-2 / \nu}=L^{-2(2-\zeta)}$, given the STS relation $\nu=1 /(2-\zeta)$.

The previuos results represent a considerable progress in the understanding of the finite-size effects but still leave us with a rather ambiguous picture for applications and analytical calculations. Important open questions are: (i) What is the dependence of $f_{c}$, and $\left\langle\left[f_{c}^{s}-\left\langle f_{c}^{s}\right\rangle\right]^{2}\right\rangle$ with the self-affine aspect-ratio parameter $k$ ? (ii) Is there a finite-size bias $\left[f_{c}-\left\langle f_{c}^{s}\right\rangle\right]$ and how does it depend on $L$ and $k$ ?. (iii) Is the RM thermodynamic limit prescription for the critical force, $\lim _{L, M=k L \zeta \rightarrow \infty}$, different from the one for extracting the RM velocity-force characteristics? In the affirmative case, how does it depend on $k$ ? (iv) Geometry and transport are closely related, as changes in the interface velocity directly affect the location of one or several geometrical crossovers [28 30]. How sensible to the value of $k$ are the geometry and the velocity of the interface? In this paper we address these open questions and show that constant force simulations in finite samples actually lead to an unambiguous thermodynamic critical force and velocity-force characteristics, which is independent of $k$, as long as $k$ is finite. We also show how the finite system transport properties scale towards the (RP) $k \rightarrow 0$ and (single particle or extreme RM) $k \rightarrow \infty$ limits as a function of $L$. Finally we discuss how our results relate to the cases of velocity-driven interfaces and other alternative methods used to define the thermodynamic critical force.

\section{MODEL, OBSERVABLES AND METHOD}

We consider the driven QEW model at zero temperature, described by

$$
\gamma \partial_{t} u(x, t)=c \partial_{x}^{2} u(x, t)+F_{p}(u, x)+f .
$$

This equation models the overdamped dynamics of the displacement field $u(x, t)$ of a one-dimensional elastic interface in a two-dimensional random medium. We will consider here a sample of size $L \times M$ with periodic boundary conditions in both directions. The pinning force derives from a bounded random potential (i.e. Random Bond disorder), $F_{p}(u, x)=-\partial_{u} U(u, x)$, with correlations

$$
\left\langle\left[U(u, x)-U\left(u^{\prime}, x^{\prime}\right)\right]^{2}\right\rangle=R\left(u-u^{\prime}\right) \delta\left(x-x^{\prime}\right),
$$

with $R(y)$ a short-ranged function of range $r_{f}$ and $\langle\cdots\rangle$ standing for the average over all disorder realizations.

In particular we study a model of Eq. (11) where the displacement field is discrete in the $x$-direction, and the random potential $U(u, x)$ is given by a sequence of uncorrelated random (Gaussian) numbers glued by a piece-wise cubic-spline with $r_{f}=1$. The details of the model are described elsewhere 31]. For each sample there is a unique critical force $f_{c}^{s}$ and a unique critical configuration $u_{c}^{s}(x)$. Both quantities are computed in an efficient and accurate way without actually solving the true dynamics 31, 32. For $f>f_{c}^{s}$, at long times, the interface acquires a steady state velocity $v^{s}$. To obtain it, we solve the dynamics of Eq. (11) from an arbitrary initial condition up to very long times using a parallel algorithm [33]. We define the width $w$ of the critical configuration:

$$
w^{2}(L, M)=\left\langle\frac{1}{L} \sum_{x=0}^{L-1}\left[u_{c}^{s}(x)-u_{c m_{c}^{s}}^{s}\right]^{2}\right\rangle,
$$

with $u_{c m_{c}}=(1 / L) \sum_{x=0}^{L-1} u_{c}^{s}(x)$ the sample dependent center of mass position of the critical configuration. When $M \sim L^{\zeta}$ it is well known that $w^{2} \sim L^{2 \zeta}$ with 33] $\zeta=1.250 \pm 0.005$.

In the following, we analyse the large size limit of (i) the critical force, (ii) the velocity for a fixed value of the external force and (iii) the width of the critical configuration, as a function of both $L$ and $k$, from the infinitely narrow sample to the infinitely wide sample.

\section{RESULTS}

\section{A. Summary of Finite-Size Scaling Results}

Here we summarize our main results. Note that in one dimension, the roughness exponent for the RM class is [33] $\zeta=1.250$, and for the RP class is [20, 34] $\zeta^{\mathrm{RP}}=1.5$. A detailed description and discussion of each result is left for the next sections. The main results are:

1. The critical force reaches a $k$-independent value $f_{c}=\lim _{L, M \rightarrow \infty}\left\langle f_{c}^{s}\right\rangle$ in the thermodynamic limit $L, M \rightarrow \infty$ for any finite $k=M / L^{\zeta}$ (see Fig. 2). This value only depends on microscopic details of the system. The velocity-force characteristics also displays the same convergence towards a unique $k$-independent thermodynamic value, $v(f)=\lim _{L, M \rightarrow \infty}\left\langle v^{s}(f)\right\rangle$. (see Fig. 10).

2. The average finite-size critical force $\left\langle f_{c}^{s}\right\rangle$ approaches the value $f_{c}$ from above if $k$ is large, and from below if $k$ is small, compared to a (nonuniversal) marginal value $k^{*} \approx 2.1 \pm 0.1$ (see Figs. 2 and [3). The asymptotic forms for this bias are well described by

$$
\left(f_{c}-\left\langle f_{c}^{s}\right\rangle\right) L^{2-\zeta} \sim\left\{\begin{aligned}
k^{1-2 / \zeta} & \text { if } k \ll k^{*} \\
-(\log k)^{1 / \delta} & \text { if } k \gg k^{*}
\end{aligned}\right.
$$




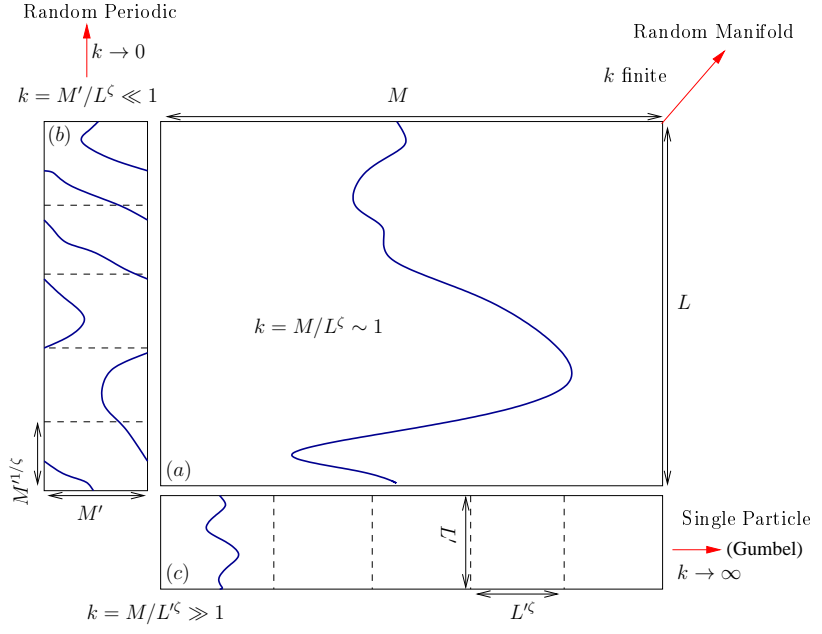

FIG. 1: (Color online) Schematic picture showing critical configurations for systems with different values of the aspect ratio $k$. In (a) the critical configuration has a width $w$ comparable to the system transverse dimension $M$. In (b), $w \gg M^{\prime}$ and the configuration wraps several times, crossing over to the RP geometry, $w \sim L^{\zeta^{\mathrm{RP}}}$. In (c) $w \ll M$ and $w / L^{\zeta}$ is a function of $k$. In the thermodynamic limit, if $k$ is kept constant, transport properties converge to a unique RM limit. If $k \rightarrow 0$ the thermodynamic limit corresponds to the RP class parametrized by the periodicity $M^{\prime}$. If $k \rightarrow \infty$ the system has a dimensional crossover towards the zero-dimensional Gumbel class (for Gaussian microscopic disorder), and the steadystate motion is static.

with $1<\delta<2$ (see Figs. 4 and 7 ), and consistent with the critical force distribution tail of the form $\ln P\left(f_{c}^{s}, L, M=k^{*} L\right) \sim-f_{c}^{s \delta}$ with $\delta=1.2 \pm 0.1$. (see Fig. 6).

3. The sample to sample fluctuations of the critical force are well described by

$\left(\left\langle f_{c}^{s^{2}}\right\rangle-\left\langle f_{c}^{s}\right\rangle^{2}\right) L^{2(2-\zeta)} \sim\left\{\begin{aligned} k^{1-\zeta^{\mathrm{RP}} / \zeta} & \text { if } k \ll k^{*} \\ (\log k)^{-2(1-1 / \delta)} & \text { if } k \gg k^{*}\end{aligned}\right.$

so they decrease with increasing $L$ or $k$ (see Figs. 5 and (8).

4. The width of the critical configuration behaves as

$$
w L^{-\zeta} \sim\left\{\begin{array}{cl}
k^{-\left(\zeta^{\mathrm{RP}} / \zeta-1\right)} & \text { if } k \ll k^{*} \\
(\log k)^{\zeta / 2 \delta} & \text { if } k \gg k^{*}
\end{array}\right.
$$

The roughness thus always increases with increasing $L$ but has a non-monotonous behaviour with $k$, decreasing for small $k$ and increasing for large $k$. Its minimum is reached for a value $k \lesssim k^{*}$. (see Fig. 9).

\section{B. The thermodynamic limit of the critical force}

The existence of a unique critical force $f_{c}$ and velocityforce characteristics $v(f)$ (see Sec. IIID) for all finite val-

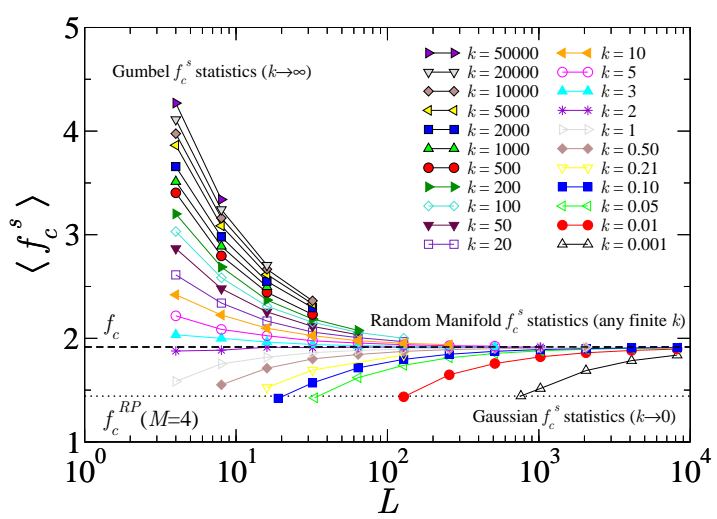

FIG. 2: (Color online) Longitudinal finite-size dependence of the averaged critical force for samples of size $L \times M$, with $M=k L^{\zeta}$. The (non-universal) thermodynamic limit $f_{c}$ attracts any finite value of $k$, thus determining this property unambiguously for the RM class. Also indicated is the (nonuniversal) thermodynamic RP critical force for a periodicity $M=4$. Note that for $k=k^{*} \approx 2$, finite-size effects are negligible for several decades of $L$.

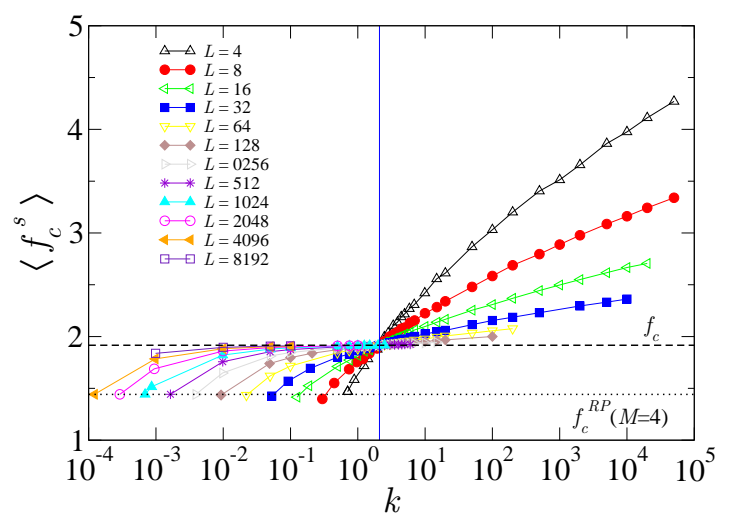

FIG. 3: (Color online) Same data as in Fig 2 but showing the transverse finite-size dependence, for each $L$, of the averaged critical force. Note the crossing at $k^{*} \approx 2$ (precisely we get $\left.k^{*}=2.1 \pm 0.1\right)$, bridging the Gaussian $(k \rightarrow 0)$ with the Gumbel $(k \rightarrow \infty)$ critical force statistics, where finite-size effects become negligible.

ues of $k$ shows that transport properties of the QEW model have an unambiguous thermodynamic limit. In other words, the infinite family of systems described by (the same) Eq. (11) but with (different) geometries parametrized by the self-affine aspect-ratio parameter $k$, is attracted to a unique RM behaviour in the large size limit (see Fig. 2). Note also that, even if $f_{c}$ and $v(f)$ are not universal, they are intrinsic; i.e., they only depend on the parameters appearing in the microscopic equation of motion. 


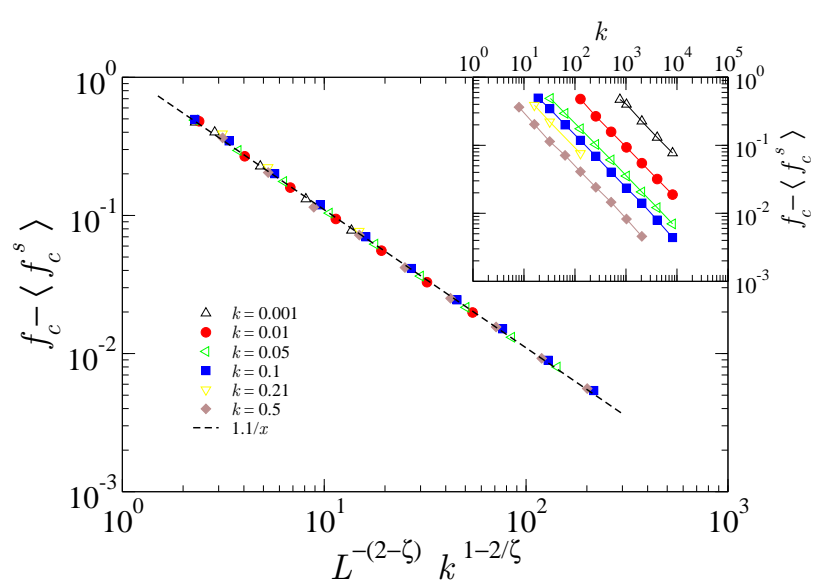

FIG. 4: (Color online) Negative finite-size bias of the critical force, $f_{c}-\left\langle f_{c}^{s}\right\rangle$, for small $k$ values. Inset: raw data. Main: scaled data. In this regime the shift is controlled exclusively by $M$ and by the RM depinning roughness exponent $\zeta$.

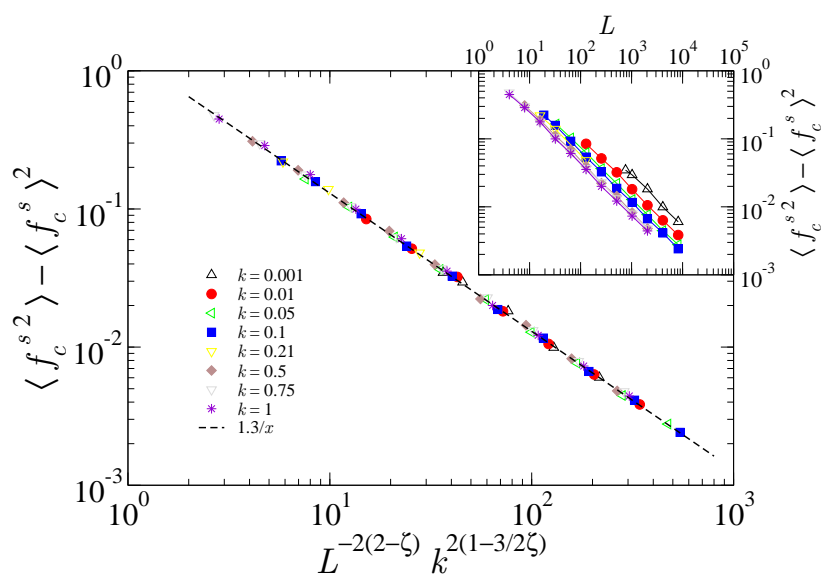

FIG. 5: (Color online) Finite-size fluctuations of the sample critical force $\left\langle f_{c}^{s 2}\right\rangle-\left\langle f_{c}^{s}\right\rangle^{2}$, for small $k$ values. Inset: raw data. Main: scaled data. In this regime the fluctuations are well described by $\left\langle f_{c}^{s 2}\right\rangle-\left\langle f_{c}^{s}\right\rangle^{2} \sim L^{-2(2-\zeta)} k^{2(1-3 / 2 \zeta)}$. Note that in order to have a non-zero $\lim _{k \rightarrow 0}\left[\left\langle f_{c}^{s 2}\right\rangle-\left\langle f_{c}^{s}\right\rangle^{2}\right]$, we need $\zeta \rightarrow 3 / 2$ in agreement to what corresponds to the (onedimensional) RP class $\left[\left\langle f_{c}^{s 2}\right\rangle-\left\langle f_{c}^{s}\right\rangle^{2}\right] \sim L^{-1}$ and roughness exponent $\zeta^{\mathrm{RP}}=3 / 2$.

\section{Finite-Size effects for small $k$}

When $k$ is much smaller than $k^{*} \sim \mathcal{O}(1)$ (i.e. $M \ll$ $\left.L^{\zeta}\right)$, the system can wrap around $M$ several times and, thus, sense the transverse periodicity of the disorder. Indeed, at a characteristic length $L_{M} \sim M^{1 / \zeta} \ll L$ the geometry of the interface crosses over from the RM to the RP class, parametrized by the periodicity $M$. This crossover is well manifested in the structure factor $\left\langle\left|u_{c}(q)\right|^{2}\right\rangle$ of the critical configuration, which displays [20], at $q \sim L_{M}^{-1}$, a crossover from the RM roughness exponent $\zeta=1.250$ (i.e., $\left.\left\langle\left|u_{c}(q)\right|^{2}\right\rangle \sim 1 / q^{1+2 \zeta}\right)$, to the RP exponent 38] $\zeta^{\mathrm{RP}}=3 / 2$ (i.e., $\left\langle\left|u_{c}(q)\right|^{2}\right\rangle \sim$
$1 / q^{1+2 \zeta^{\mathrm{RP}}}$ ) increasing the observation lengthscale $q^{-1}$.

When $L$ grows with fixed $k, L_{M}$ grows as $L_{M} \sim k^{1 / \zeta} L$. Since the average critical force $\left\langle f_{c}^{s}\right\rangle$ and velocity $\left\langle v^{s}\right\rangle$ for a finite system are determined by the typical behaviour at small length-scales $\left(l<L_{M}\right)$ or short wavelength modes, the critical force must approach the RM thermodynamic value $f_{c}$ as $L_{M}$ grows, even when the large scale geometry $\left(l>L_{M}\right)$ is still described by $\zeta^{\mathrm{RP}}$ instead of $\zeta$. Since for our model of Eq. (1), the RP critical force $f_{c}^{\mathrm{RP}} \simeq \lim _{L \rightarrow \infty}\left\langle f_{c}^{s}(L, M)\right\rangle$ with $M \sim 1$ is always smaller that the RM critical force $f_{c}$, for small $k$ the thermodynamic limit is approached from below, as can be observed in Figs. 2]and 3. Furthermore, we can see in Fig. 4 a negative finite-size bias of the critical force $f_{c}-\left\langle f_{c}^{s}\right\rangle$ for small values of $k$ that smoothly follows Eq. (4).

Let us introduce an heuristic argument to understand the scaling. In principle, one can think the string as being composed by $L / L_{M}=k^{-1 / \zeta} \gg 1$ "RM blocks", of longitudinal size $L_{M}$ and transverse size $M$. Note that each of these blocks has precisely the "proper" aspect-ratio $L_{M}^{\zeta} / M=1$. If we consider that each of these blocks participates in the total critical force with independent contributions, such that they average to $\overline{f_{M}}$ with a dispersion $\sigma_{M}$, where $\cdots$ stands for an average over the independent RM blocks, then we can write:

$$
\begin{array}{r}
\left\langle f_{c}^{s}(L, M)\right\rangle \approx \overline{f_{M}} \\
\left\langle f_{c}^{s^{2}}(L, M)\right\rangle-\left\langle f_{c}^{s}(L, M)\right\rangle^{2} \sim \frac{\sigma_{M}^{2}}{L / L_{M}},
\end{array}
$$

The above assumptions are consistent with the fact that $f_{c}^{s}$ has almost a Gaussian statistics [19] if $M \ll L^{\zeta}$, by virtue of the central limit theorem for the sum of many pinning forces with finite variance, which are uncorrelated at distances smaller than $L_{M}$. If $\overline{f_{M}}$ represents minus the average pinning force on a given block of size $L_{M} \times M$, then we can write $\overline{f_{M}} \sim f_{c}^{s}\left(L_{M}, M\right)$. Since the interface in each block is, by definition, in the RM regime, we can write $\sigma_{M} \sim L_{M}^{\zeta-2}$ for its sample to sample fluctuations. We thus get:

$$
\begin{array}{r}
\left\langle f_{c}^{s}(L, M)\right\rangle \approx\left\langle f_{c}^{s}\left(L_{M}, M\right)\right\rangle \\
\left\langle f_{c}^{s^{2}}(L, M)\right\rangle-\left\langle f_{c}^{s}(L, M)\right\rangle^{2} \sim k^{2(1-3 / 2 \zeta)} L^{-2(2-\zeta)}
\end{array}
$$

First, let us note that the predicted finite-size scaling dependence on $L$ and $k$ for $\left\langle f_{c}^{s^{2}}(L, M)\right\rangle-\left\langle f_{c}^{s}(L, M)\right\rangle^{2}$ is indeed what we observe in Fig. 55. Second, let us note that $\left\langle f_{c}^{s}\right\rangle$ depends only on $M$ (as $L_{M}=M^{1 / \zeta}$ ), in consistency with the behaviour shown in Fig 4 . If this bias scales with the longitudinal size in the same way as the sample to sample fluctuations, one can predict $\left[f_{c}-\left\langle f_{c}^{s}(L, M)\right\rangle\right] \approx$ $\left[f_{c}-\left\langle f_{c}^{s}\left(L_{M}, M\right)\right\rangle\right] \sim L_{M}^{\zeta-2} \sim M^{2 / \zeta-1} \sim L^{-(2-\zeta)} k^{(1-2 / \zeta)}$, as shown in Fig. 4 .

It is interesting to note that in order to have $\quad \lim _{k \rightarrow 0}\left[\left\langle f_{c}^{s^{2}}(L, M)\right\rangle-\left\langle f_{c}^{s}(L, M)\right\rangle^{2}\right] \sim$ $k^{2(1-3 / 2 \zeta)} L^{-2(2-\zeta)}$ finite in the $L \rightarrow \infty$ thermodynamic limit, which corresponds to the RP class with a fixed periodicity $M$, we require that $\zeta \rightarrow \zeta^{\mathrm{RP}}=3 / 2$. 


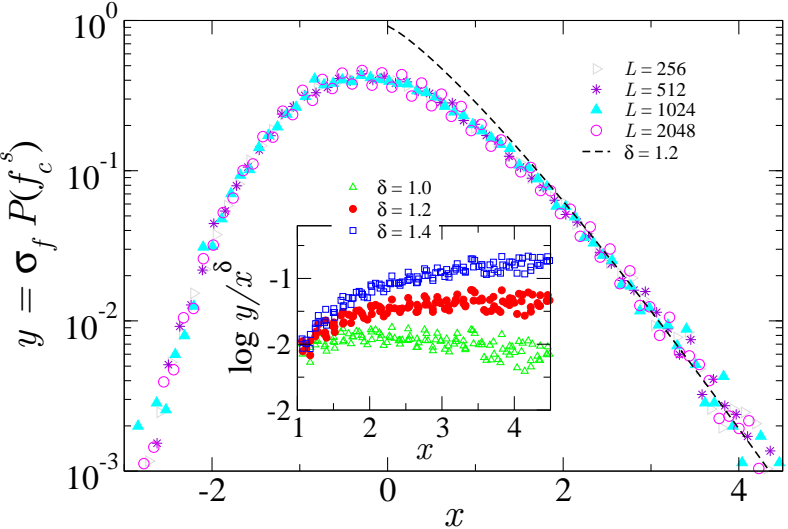

FIG. 6: (Color online) Scaled distribution function for the critical force, $y=\sigma_{f} P\left[f_{c}^{s}(k=2)\right]$ against $x=\left(f_{c}^{s}-\left\langle f_{c}^{s}\right\rangle\right) / \sigma_{f}$, with $\sigma_{f}=\sqrt{\left(\left\langle f_{c}^{s}{ }^{2}\right\rangle-\left\langle f_{c}^{s}\right\rangle^{2}\right)}$. Different system sizes are shown, $L \times M$ with $M=k L^{\zeta}$ for $k=2$. The large $f_{c}^{s}$ tail can be fitted to a stretched exponential, $P\left(f_{c}^{s}\right) \sim \exp \left[-f_{c}^{s \delta}\right]$, characterized by $\delta=1.2 \pm 0.1$, as can be observed in the inset.

Therefore, $\left\langle f_{c}^{s^{2}}(L, M)\right\rangle-\left\langle f_{c}^{s}(L, M)\right\rangle^{2} \sim L^{-1}$, again consistent with the prediction for the critical force fluctuations in the RP class.

\section{Finite-Size effects for large $k$}

When $k$ is large, periodicity effects disappear, since the critical configuration cannot wind around the cylinder of perimeter $M$. In turn, we start to observe extreme value statistics effects. As described in [19], in the $k \rightarrow \infty$ limit the critical force distribution tends to a Gumbel function; i.e., the critical force of each sample can be thought as the maximum of independent identically distributed (iid) variables with a (stretched) exponential-tailed distribution. This explains why $\left\langle f_{c}^{s}(L, M)\right\rangle$ approaches $f_{c}$ from above in Fig. 2 Indeed, considering $\left\langle f_{c}^{s}\right\rangle$ as the maximum among the critical forces of many independent configurations we can expect a growth with increasing $M$ at $L$ fixed, which is observed in Fig. 3. Since metastable (or quasi-critical) configurations just below (or above) the depinning transition are essentially decorrelated in a distance of the same order than its width $w \simeq L^{\zeta}$, the number of such independent random variables is precisely $k=M / L^{\zeta}$.

Let us analyse the case when $k$ is finite and close to $k^{*}$. On one hand, in this case finite size effects are less pronounced, as shown in Fig. 2. We can expect that, in the same sense that $f_{c}^{s}$ is attracted to $f_{c}$, the distribution function should also be attracted to a thermodynamic limit which would be close to the one with $k=k^{*}$. Note that the critical force $f_{c}^{s}$ of a system of size $L \times k^{*} L^{\zeta}$ is distributed according to a function which is intermediate between the Gaussian and the Gumbel's [19]. Although when the shape of this function is not known analytically, we know that it must decay faster than a power
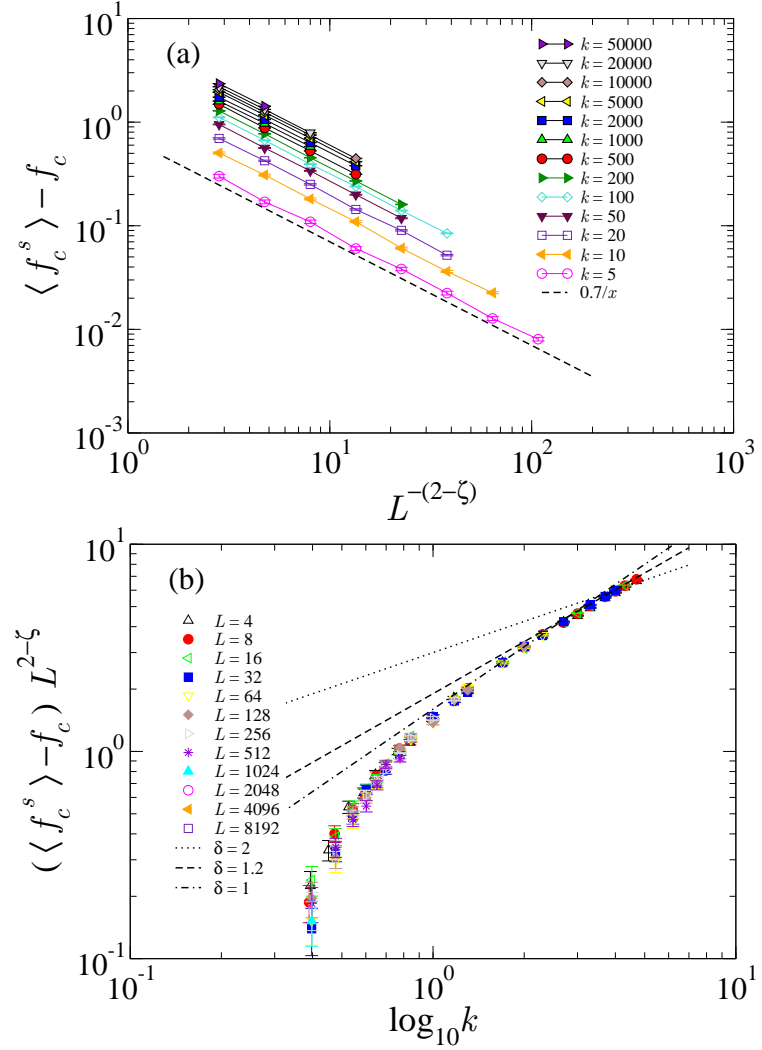

FIG. 7: (Color online) Positive finite-size shift of the critical force, $f_{c}-\left\langle f_{c}^{s}\right\rangle$, for large $k$. (a) The shift decays as $L^{-(2-\zeta)}$, with a $k$-dependent prefactor. (b) As expected from extreme value statistics, at the largest $k$ the shift increases with $k$ logarithmically, $f_{c}-\left\langle f_{c}^{s}\right\rangle \sim(\log k)^{1 / \delta}$, with $\delta=1.2$ in agreement with the tail exponent obtained in Fig. 6. We compare with $\delta=2$ expected for the maximum of iid Gaussian random variables and $\delta=1$ for for the maximum of iid exponential variables.

law, since the maximum among $k=M / L^{\zeta}$ of such systems is attracted to the Gumbel class in the $k \rightarrow \infty$ limit. To support this idea, let us consider the tail of $P\left(f_{c}^{s}\right)$ described in particular as a stretched exponential decay, $\ln P\left(f_{c}^{s}\right) \sim-f_{c}^{s \delta}$, with $1 \leq \delta \leq 2$ (the bounds corresponding to the Gaussian case, $\delta=2$, and to the Gumbel case, $\delta=1)$. In order to test this idea, we show in Fig. 6 the distribution function for the sample critical force $f_{c}^{s}$ corresponding to $k=2$ where the system size effects are almost negligible. It is shown in this case that the tail exponent characterizing the stretched exponential behaviour is $\delta=1.2 \pm 0.1$.

Therefore, based on the observed stretched exponential behaviour and from standard extreme value statistics arguments [35] we get that the average of $f_{c}^{s}=$ $\max \left\{f_{1}^{(0)}, f_{1}^{(1)}, \ldots, f_{1}^{(k)}\right\}$ should grow as $\left\langle f_{c}^{s}\right\rangle-f_{c} \sim$ $L^{-(2-\zeta)}(\log k)^{1 / \delta}$. Here we have used again that the finite size bias for a $L \times L^{\zeta}$ (or $k \sim 1$ ) system behaves as the sample to sample fluctuations $\left\langle f_{1}\right\rangle-f_{c} \sim L^{-(2-\zeta)}$ in the $L \rightarrow \infty$ limit. This prediction is consistent to 

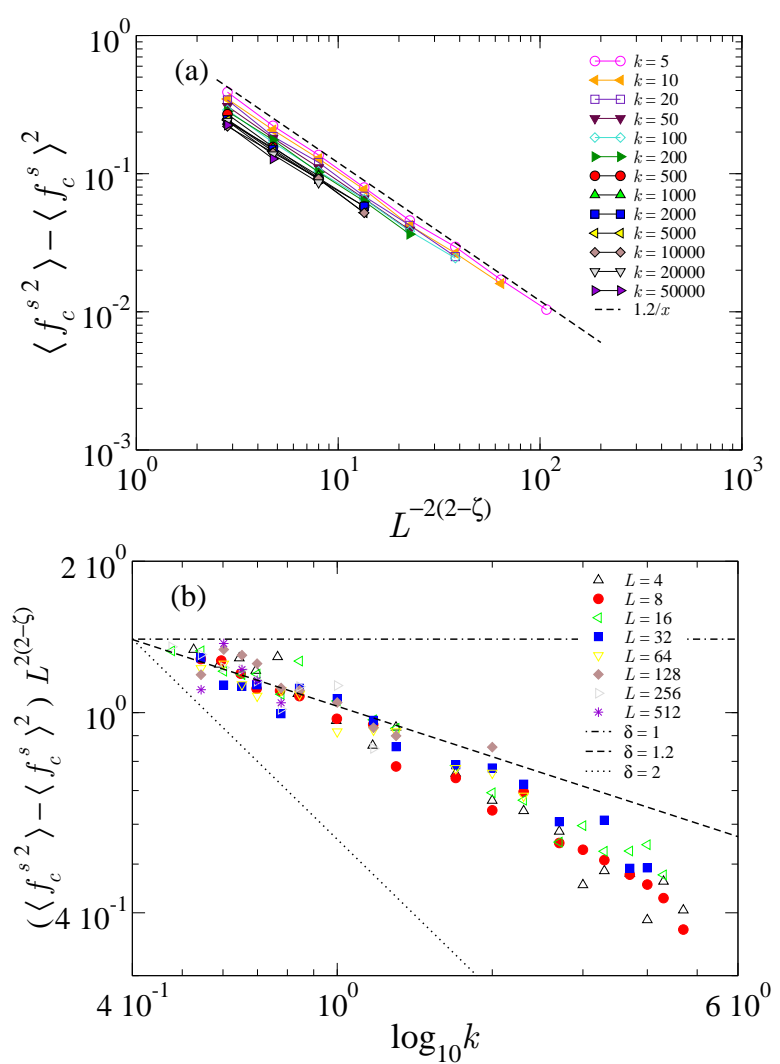

FIG. 8: (Color online) Finite-size fluctuations of the critical force $\left\langle f_{c}^{s 2}\right\rangle-\left\langle f_{c}^{s}\right\rangle^{2}$, for large $k$. (a) $\left\langle f_{c}^{s 2}\right\rangle-\left\langle f_{c}^{s}\right\rangle^{2} \sim L^{-2(2-\zeta)}$, same as in the low $k$ regime (Fig. 5). (b) Dependence with $k$. We find $\left\langle f_{c}^{s 2}\right\rangle-\left\langle f_{c}^{s}\right\rangle^{2} \sim(\log k)^{-2(1-1 / \delta)}$, consistent with the finite-size shift expected from extreme statistics. Note the consistency with $\delta=1.2$ from Fig. 7

what is found in the simulations, as shown in Fig. 7 The large $k$ behaviour is consistent with the obtained value of the tail exponent $\delta=1.2$. Within this picture, standard extreme value arguments also predict $\left\langle f_{c}^{2}\right\rangle-\left\langle f_{c}^{s}\right\rangle^{2} \sim L^{-2(2-\zeta)}(\log k)^{-2(1-1 / \delta)}$. This is also consistent with Fig. 8, where we compare the prediction using the same value of $\delta$ obtained in Fig 6. As it can be observed in Fig. 8(b), in the large $L$ limit the data is consistent with the value of $\delta=1.2 \pm 0.1$, ruling out the bounds $\delta=2$ for the maximum of iid Gaussian variables, and $\delta=1$ for the maximum of iid exponential variables.

It is interesting to note that since $\delta>1$ the sample to sample fluctuations of the critical force decrease for increasing $k$, unlike the mean value of the critical force, which increases with $k$. Therefore, the growing sample critical force reaches a sharply defined value in the large $k$ limit. This might be important for experiments.
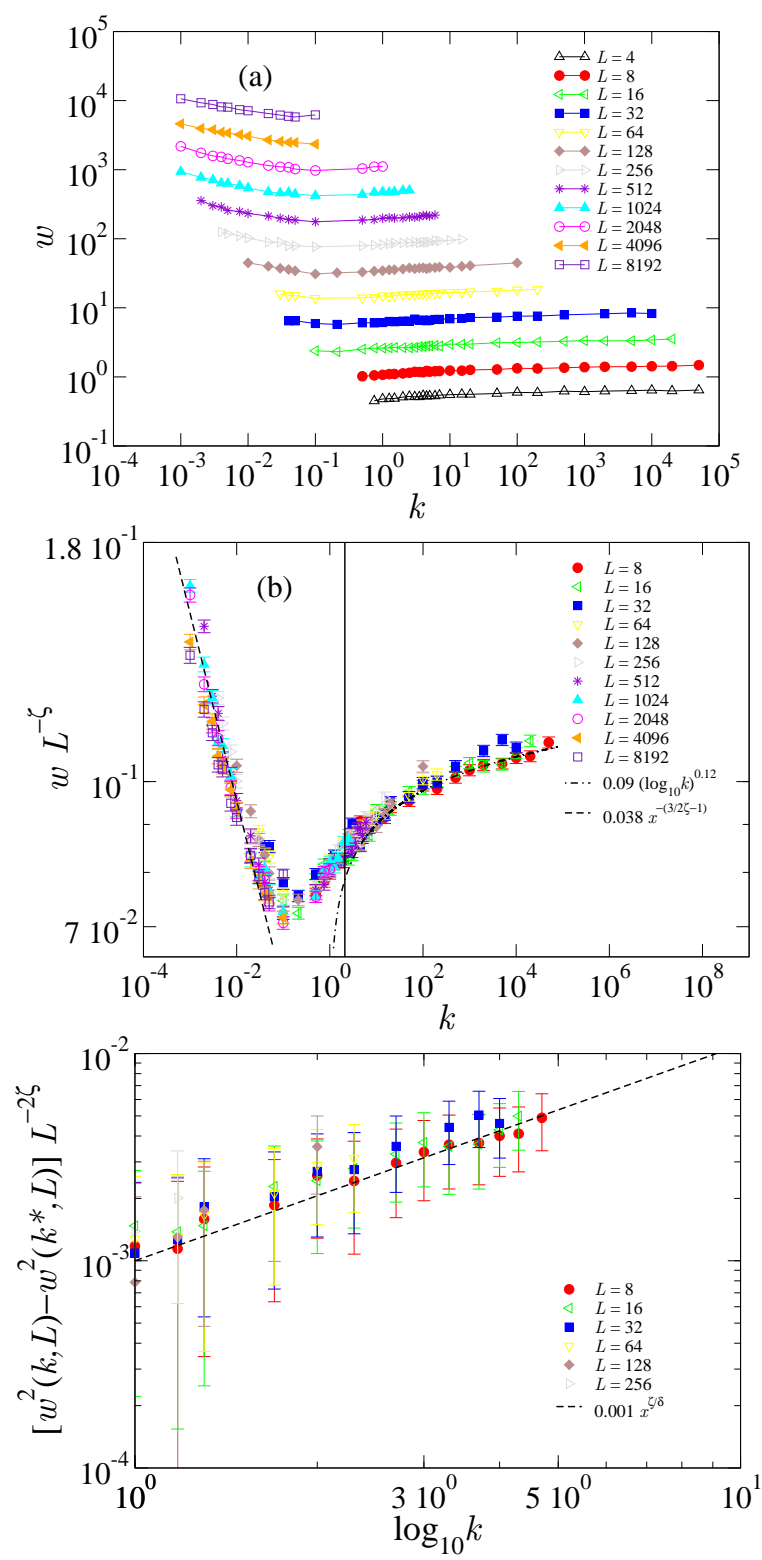

FIG. 9: (Color online) Anisotropic finite-size analysis of the width $w$ of the critical configurations. (a) Raw-data. (b) Scaled data, according to $w \sim L^{\zeta}$, vs $k$. In the low $k$ regime, $w \sim L^{\zeta} k^{-\left(\zeta^{\mathrm{RP}} / \zeta-1\right)}$ (dashed-line). Note that for a non-zero $\lim _{k \rightarrow 0} w$ we need $\zeta \rightarrow \zeta^{\mathrm{RP}}=3 / 2$, corresponding to the (onedimensional) RP class (Compare with the critical-force fluctuations in the same regime shown in Fig. 4). The width for large $k$ is roughly described by some power-law of $\log k$ (dotdashed line). The solid line indicates $k^{*}=2.1$. (c) Scaled data, according to $\left[w^{2}(k, L)-w^{2}\left(k^{*}, L\right)\right] \sim L^{2 \zeta}(\log k)^{\zeta / \delta}$ and using the values $\zeta=1.250$ and $\delta=1.2$, showing the agreement between the data and the scaling prediction.

\section{Finite-size effects in the roughness of the critical configuration}

The typical interface global width or roughness also manifests size effects at depinning, which are consistent 
with the finite-size scaling for the critical force.

Let us first describe the low- $k$ behaviour of the width $w$ shown in Fig 9. From the study of the RM to RP crossover at $L_{M} \sim M^{1 / \zeta} \ll L$ we know that [20] $w(L) \sim$ $L_{M}^{\zeta}\left(L / L_{M}\right)^{\zeta^{\mathrm{RP}}}$. We thus get

$$
w(L) \sim k^{-\left(\zeta^{\mathrm{RP}} / \zeta-1\right)} L^{\zeta}
$$

in consistency with the asymptotic behaviour shown in Fig. 9(b) for small values of $k$, where $\zeta^{\mathrm{RP}}=3 / 2$ for our one-dimensional case.

Turning now to the behaviour of the roughness critical configurations at large values of $k$, we can see that, interestingly, it displays an approximate logarithmic growth with $k$, as shown in Fig 9(b). This was already observed in Ref. [24]. Here we link such behaviour with the critical force statistics and predict its scaling form. We start by noting that in order to have a logarithmic growth of $f_{c}^{s}$ with $k$ in this regime, either the individual pinning forces on the monomers of the critical configuration get more correlated in order to increase $f_{c}^{s}$, or they remain uncorrelated but acquire an enhancement of their dispersion with increasing $k$. Since we do not observe increased correlations between the individual pinning forces acting on the monomers of $u_{c}$ for large $k$, the last scenario is the most plausible. A logarithmic enhancement in the prefactor $w^{2} / L^{\zeta}$ can be thus heuristically understood as follows. From the Larkin formulation we can define effective Larkin length $L_{c} \sim$ $\left(f_{c} / c r_{f}\right)^{-1 / 2}$ and roughness $w \approx r_{f}\left(L / L_{c}\right)^{\zeta}$. Extending this idea to sample to sample fluctuations, we consider that $\left\langle L_{c}^{s}(k, L)\right\rangle \sim\left(\left\langle f_{c}^{s}\right\rangle / c r_{f}\right)^{-1 / 2}$ and $w \approx r_{f}\left(L /\left\langle L_{c}^{s}\right\rangle\right)^{\zeta}$. Therefore, using that $\left\langle f_{c}^{s}\right\rangle-f_{c} \sim(\log k)^{1 / \delta}$ we easily get $w(k, L) / L^{\zeta} \sim(\log k)^{\zeta / 2 \delta}$ in the very large $k$ limit, where $\left\langle f_{c}^{s}\right\rangle \gg f_{c}$. Since our data does not reach such limit, we can not neglect the $f_{c}$ contribution. A corrected version reads $\left(w(k, L) / L^{\zeta}\right)^{2}-\left(w\left(k^{*}, L\right) / L^{\zeta}\right)^{2} \sim(\log k)^{\zeta / \delta}$, where we have used the $k$-independent thermodynamic limit $\lim _{L \rightarrow \infty} w(k, L) / L^{\zeta} \approx w\left(k^{*}, L\right) / L^{\zeta} \sim r_{f} L_{c}^{-\zeta}$. Fig. 9(c) presents a scaled version of the data to test this idea, where we have used that $\zeta=1.250, \delta=1.2$ (see fit in Fig. (7) and $k^{*} \approx 2$ (see Fig. 3), and shows a very good agreement with the scaling prediction.

In summary, the increasingly rare critical configurations for large $k$ can be thus seen as being pinned by an effectively stronger uncorrelated microscopic disorder keeping the same elasticity and microscopic disorder correlator range. In other words, extreme statistics shorten the effective Larkin length on those critical configurations.

\section{Finite-size effects in the velocity-force characteristics}

When $f>f_{c}^{s}$, the elastic interface moves steadily with velocity $v^{s}(f)$ and the geometry displays a crossover in the roughness from the exponent $\zeta$ to the exponent $\zeta^{\text {th }}=$

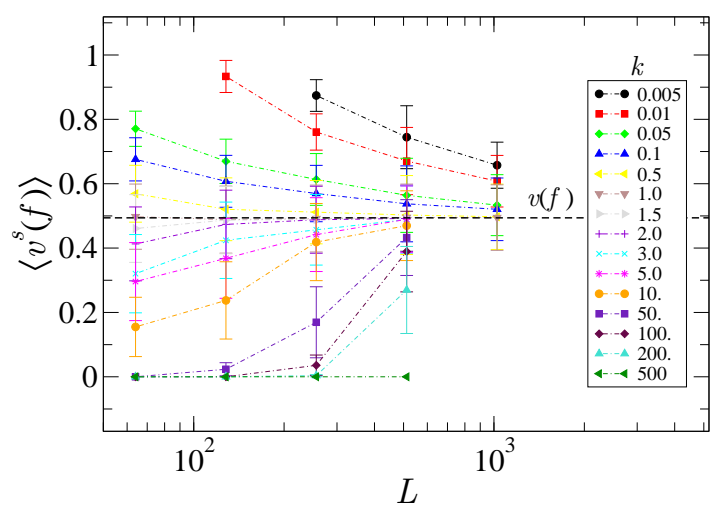

FIG. 10: (Color online) Finite size effects in the mean velocity for different values of the self-affine aspect ratio $k$, and the same driving force $f=1.95>f_{c}$. We observe that velocity curves for finite $k$ values are attracted to the same limit $v(f) \approx 0.5$, except for $k=500$, where rare blocking configurations still dominate and $f_{c}^{s}>f$ for the range of $L$ values analysed. The black dashed line indicates the estimation of the stationary velocity in the thermodynamic limit $\left.\lim _{L \rightarrow \infty}\left\langle v^{s}(f)\right\rangle\right|_{k} \rightarrow v(f)$.

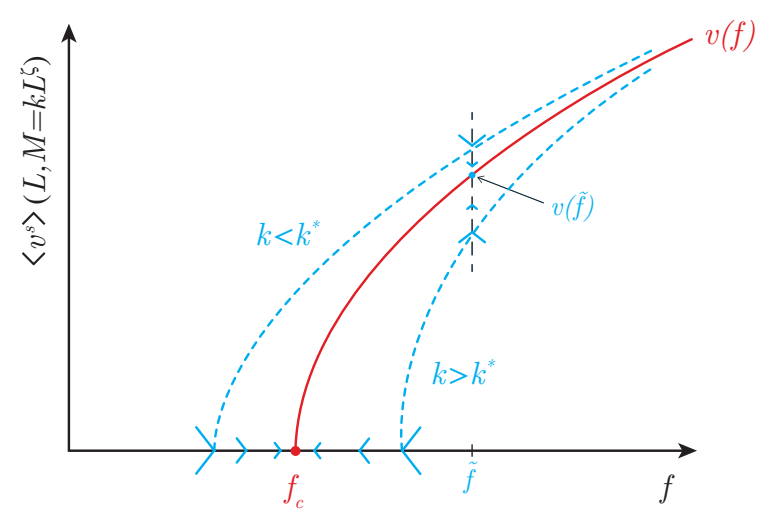

FIG. 11: (Color online) Schematic $k$-dependence of the velocity-force characteristics. For $k>k^{*}$ the critical force bias is positive, $f_{c}^{s}<f_{c}$, and therefore the velocity at a given force $f>f_{c}$ approaches the thermodynamic limit from below, $\left\langle v^{s}(f)\right\rangle<v(f)$. The opposite is observed when $k<k^{*}$ : the critical force bias is negative and hence the velocity is $\left\langle v^{s}(f)\right\rangle>v(f)$.

$(2-d) / 2$ at the characteristic scale $\xi \sim v^{-\nu / \beta}$. Given the presence of this extra length-scale depending on the force excess, it is not obvious whether the same thermodynamic limit prescription for the RM critical force (i.e., to fix the aspect-ratio parameter $k=M L^{-\zeta}$ ) will work for the velocity, yielding a unique $k$-independent velocity limit $v(f)$.

In Fig. 10 we show that the prescription for $f_{c}$ works well for $v(f)$. We observe that a wide range of values of $k$ tends to converge to a $k$-independent, force dependent steady-state velocity, $\left.\lim _{L \rightarrow \infty}\left\langle v^{s}(f ; k, L)\right\rangle\right|_{k} \rightarrow v(f)$. At finite $L$, we observe that $\left\langle v^{s}(f)\right\rangle>v(f)$ for small $k$, and $\left\langle v^{s}(f)\right\rangle<v(f)$, for large $k$. This is consistent with 


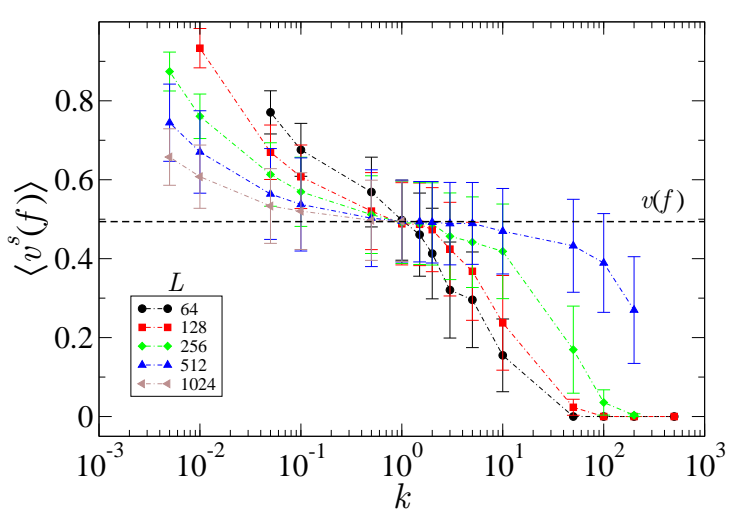

FIG. 12: (Color online) Same data as in Fig. 10 but showing the transverse finite-size dependence, for each L, of the averaged stationary velocity. Note the crossing at $k_{v}^{*}$, where finitesize effects become negligible, bridging the Gaussian $(k \rightarrow 0)$ with the Gumbel $(k \rightarrow \infty)$ statistics.

the behaviour of the finite-size average critical force (see schema in Fig. 11): $f_{c}^{s}$ is biased to greater values of $f$ as $k$ increases above $k^{*}$, so, if we assume a monotonous and continuous behaviour of the velocity-force curve, for a given $f$ the velocity average $\left\langle v^{s}(f)\right\rangle$ should be smaller as $k>k^{*}$ increases. For very large $k$ indeed, we can be in the situation where $f\left\langle f_{c}^{s}\right.$ and thus $\left\langle v^{s}\right\rangle=0$, as is seen in Fig. 10 for $k=500$. On the other hand, for small values of $k<k^{*}, f_{c}^{s}$ decreases with $k$, and at a fix force $f$ the average velocity will be larger as $k$ decreases. This is why curves for $k<k_{v}^{*} \simeq 1 \sim k^{*}$ converge from above in Fig. 10 to the thermodynamic limit.

In Fig. 12 we observe the behavior of $\left\langle v^{s}(f)\right\rangle$ as a function of $k$ for different system sizes. For the working force $f=1.95>f_{c}$, a crossing of all curves at $k_{v}^{*} \simeq 1$ can be observed, but also, how curves are apparentely atracted to a unique constant value, both avobe and below $k_{v}^{*}$, as $L$ increases. We find that $k_{v}^{*} \sim \mathcal{O}(1)$, without appreciably varying with $f$. This shows that the same critical force prescription is adequate to obtain the thermodynamic limit of the velocity-force curve, and that finite size effects at any finite $k$, vanish as $L \rightarrow \infty$.

\section{DISCUSSION}

\section{A. Comparison with the velocity-driven ensemble}

In this paper we have defined the critical force of a one-dimensional QEW line in a given finite disorder sample of dimensions $L \times M$ with periodic boundary conditions in all directions, driven by a uniform, constant force $f$. In some situations however, the interface is velocitydriven in a infinitely wide medium, and the driving force $f$ is replaced by a term $m^{2}[v t-u(x, t)]$, with $v$ the imposed mean velocity. We will argue that the results of Eqs. (4), and (5) are also relevant for this case, and that a close connection exists simply by relating the curva- ture parameter $m$ and the transverse periodic dimension $M$. Since the parabolic drive sets a characteristic lengthscale $L_{m} \sim 1 / m$ in the longitudinal direction, we can compare it directly with the length $L_{M} \sim M^{1 / \zeta}$ set by the periodic boundary conditions in the constant force simulations. We can hence relate $M^{1 / \zeta}$ and $1 / m$, so the limit of small $m$ corresponds to the large $M$ limit, and viceversa.

The critical force is defined in the quasistatic limit of the velocity-driven interface as $\left\langle f_{c}^{s}(L, m)\right\rangle \equiv$ $\lim _{v \rightarrow 0+} m^{2}\langle[v t-u(x, t)]\rangle$ for stationary values of $u(x, t)$ It can be compared with the critical force $\left\langle f_{c}^{s}(L, M)\right\rangle$ discussed in the previous subsections. Functional Renormalization group (FRG) calculations predict when $L \rightarrow \infty$ that $\left\langle f_{c}^{s}(m)\right\rangle=f_{c}+c_{1} m^{2-\zeta}$ in the small $m$ limit, with $c_{1}$ a negative constant and $f_{c}$ the thermodynamic critical force. If we assume $L$ very large and define $k_{m}=(L m)^{-\zeta}$, such prediction reads $\left\langle f_{c}^{s}(m)\right\rangle=f_{c}+$ $c_{1} k_{m}^{1-2 / \zeta} L^{\zeta-2}$. As shown in Eq. 4 this is exactly the same scaling we find for $\left\langle f_{c}^{s}\left(L, M=k L^{\zeta}\right)\right\rangle$ for small $k$, with $f_{c}>\left\langle f_{c}^{s}(m)\right\rangle$ assured by the FRG prediction $c_{1}<0$. This supports our identification of $k$ with $k_{m}$, and we can expect Eqs. (4) and (5) to hold in the velocity-driven ensemble by replacing $k \mapsto k_{m}$.

To further emphasize the connection we note that the numerical extrapolation of $\left\langle f_{r}^{s}(m)\right\rangle$ in the velocitydriven ensemble yield a value [36] $f_{c} \sim 1.9$, indistinguishable from ours, $f_{c} \approx 1.916 \pm 0.001$, for the same microscopic disorder. On the other hand, the prediction $\left\langle f_{c}^{s}(m)\right\rangle=f_{c}+c_{1} k_{m}^{1-2 / \zeta} L^{\zeta-2}$ shows that for small $k_{m}$ (i.e. large $m$ compared to $L^{-1}$ ), the critical force is smaller than $f_{c}$, as we see in Fig. 4 for small $k$. Moreover, as shown in Fig. 2 of Ref. [36], when $k_{m}$ is large (i.e., $m$ small compared to $\left.L^{-1}\right), f_{c}^{s}(m)$ can become larger than the extrapolated $f_{c}$. This is due to extreme value statistic effects similar to the ones discussed in the previous sections: as the curvature of the parabola vanishes for a fixed $L$, the interface can get blocked in more rare configurations with systematically higher critical forces.

In summary, the transport properties have a unique limit and similar finite-size effects in the two ensembles. Only the roughness of the critical configurations for small $k$ or $k_{m}$ are different, since for the velocity-driven case, the roughness beyond the length-scale $L_{m} \sim 1 / m$ crosses over from $\zeta \approx 1.25$, to $\zeta^{\mathrm{m}}=0$ (instead of $\zeta^{\mathrm{RP}}=3 / 2$ ), so $w \sim m^{-\zeta}$. On the other hand, for small $m$, such that $m L \ll 1$, we expect to observe a behaviour analogous to Eq. (6) for large $k$. This has not been analyzed yet in the velocity-driven ensemble.

Finally, it is worth mentioning that an original alternative approach was analytically implemented in Ref. [23], by defining a critical force for a fixed center of mass position. This choice avoids rare configurations as the interface can not explore the disorder in the transverse directions beyond the length-scale set by its own width $w \sim L^{\zeta}$. It is thus equivalent to work with a systemsize satisfying $k \sim 1$ ( or $k_{m} \sim 1$ ), and must thus have the same unique thermodynamic limit. The advantage 
of this method is that it is parameter free, and size effects are only controlled by $L$. In addition, it does not present crossovers, and the critical configuration geometry always belong to the (non-extreme) RM class. This method has been so far only implemented analytically however.

\section{B. Implications for the non-steady universal relaxation of the velocity}

It is interesting to relate the finite-size bias of the critical force, Eq. (4), with the universal non-steady relaxation at the thermodynamic depinning threshold [37]. In Ref. 33] it was noted that the short-time relaxation of an initially flat interface at the $R M$ thermodynamic critical force $f_{c}$ can be effectively described as an interface of "size" $\ell(t)$ which is quasistatically driven by the finite-size bias of the critical-force. That is, we assume that $v(t)$ instantaneously satisfies the steadystate relation $v(t) \sim\left[f_{c}-f_{c}(\ell(t))\right]^{\beta}$, where the effective "size" grows with time as the growing correlation length $\ell(t) \sim t^{1 / z}$, with $z$ the dynamical exponent. By assuming $f_{c}-f_{c}(\ell(t)) \sim \ell(t)^{\zeta-2}$, we get the critical relaxation $v(t) \sim t^{-\beta / \nu z}$

The finite size scaling of Eq. (4) allows us now to better justify the above assumptions. The initially flat relaxing string of size $L$, in the small (non-steady) velocity limit such that the adiabatic approximation holds, effectively becomes a (pseudo) critical configuration confined in a system of effective size $L \times w(t)$. This situation is equivalent to the one described in Sec IVA with $m(t) \sim$ $w(t)^{-1 / \zeta}$, in the quasistatic drive limit. This defines an effective aspect-ratio parameter $k(t) \sim w(t) / L^{\zeta} \ll k^{*}$, and allow us to write,

$$
\left.v(t) \sim\left[f_{c}-f_{c}(L, k(t))\right)\right]^{\beta} \sim k(t)^{\beta(1-2 / \zeta)} \sim t^{-\beta / \nu z},
$$

where in the second term we have used the $k \ll k^{*}$ scaling for the bias of the critical force, Eq. (4), and in the third term the STS relation $\nu=1 /(2-\zeta)$. When $k(t) \sim k^{*}$ the bias vanishes, corresponding to the vanishing of the velocity in a finite system when [37] $\ell(t) \sim L$. The string is then blocked by a typical RM critical configuration. In order to explore rare critical configurations we need to drive the system above the thermodynamic critical force $f>f_{c}$. Then, from Eq. (4), and the same adiabatic approximation for $v(t)$, we can expect a crossover to a new regime in the non-steady relaxation, from a powerlaw to a slower logarithmic decay.

\section{CONCLUSIONS}

We have shown that there exists a unique, unambiguous thermodynamic limit for the transport properties of driven elastic interfaces in random media, irrespective of the precise relation between the longitudinal and transverse dimensions of the system, only provided they maintain a definite scaling relation in the large size limit. Namely, any finite value of the self-affine aspect-ratio parameter $k=M / L^{\zeta}$, with $\zeta \simeq 1.250$ the depinning exponent, leads to exactly the same transport properties in the large-size limit. We have also characterized in details the finite-size effects in the critical force fluctuations for small and large values of $k$. Our results thus extends the one of Ref. [19] in several useful ways. In particular, we show that the thermodynamic critical force is not only finite if $k$ is finite, but that it is independent of $k$; i.e., it is unique. We also report a finite-size bias or shift in the critical force, which was unnoticed before, as, in general, only reduced variables (of zero mean) were analyzed. We give good evidences that the velocity-force characteristics is itself a unique curve in the thermodynamic limit, and interpret it as an attractor for the stationary and even non-stationary behavior of any finite system with well defined geometry. Finally, we have also shown that our results are completely consistent with the ones obtained for velocity-driven interfaces, where $f \rightarrow m^{2}(v t-u(x, t))$, so the two ensembles have the same transport properties in the thermodynamic limit and are thus equivalent.

\section{Acknowledgements}

A.B.K. and A.R. acknowledge partial support by the France-Argentina MINCYT-ECOS A12E05. E.E.F. acknowledges partial support by the FranceArgentina Bernardo Houssay Program 2012. S.B. acknowledges partial support by the France-Argentina MINCYT-ECOS A12E03. Partial support from Project PIP11220090100051 (CONICET) and Project PICT2010/889 are also acknowledged.
[1] S. Lemerle, J. Ferré, C. Chappert, V. Mathet, T. Giamarchi, and P. Le Doussal, Phys. Rev. Lett. 80, 849 (1998).

[2] M. Bauer, A. Mougin, J. P. Jamet, V. Repain, J. Ferré, R. L. Stamps, H. Bernas, and C. Chappert, Phys. Rev. Lett. 94, 207211 (2005).

[3] M. Yamanouchi, D. Chiba, F. Matsukura, T. Dietl, and H. Ohno, Phys. Rev. Lett. 96, 096601 (2006).
[4] P. J. Metaxas, J. P. Jamet, A. Mougin, M. Cormier, J. Ferré, V. Baltz, B. Rodmacq, B. Dieny, and R. L. Stamps, Phys. Rev. Lett. 99, 217208 (2007).

[5] J.-C. Lee, K.-J. Kim, J. Ryu, K.-W. Moon, S.-J. Yun, G.-H. Gim, K.-S. Lee, K.-H. Shin, H.-W. Lee, and S.-B. Choe, Phys. Rev. Lett. 107, 067201 (2011).

[6] P. Paruch, T. Giamarchi, and J. M. Triscone, Phys. Rev. Lett. 94, 197601 (2005). 
[7] P. Paruch and J. M. Triscone, Appl. Phys. Lett. 88, 162907 (2006).

[8] J. Y. Jo, S. M. Yang, T. H. Kim, H. N. Lee, J.-G. Yoon, S. Park, Y. Jo, M. H. Jung, and T. W. Noh, Phys. Rev. Lett. 102, 045701 (2009).

[9] P. Paruch, A. B. Kolton, X. Hong, C. H. Ahn, and T. Giamarchi, Phys. Rev. B 85, 214115 (2012).

[10] J. Guyonnet, E. Agoritsas, S. Bustingorry, T. Giamarchi, and P. Paruch, Phys. Rev. Lett. 109, 147601 (2012).

[11] S. Moulinet, A. Rosso, W. Krauth, and E. Rolley, Phys. Rev. E 69, 035103(R) (2004).

[12] Le Doussal, P., Wiese, K. J., Moulinet, S., and Rolley, E., EPL 87, 56001 (2009).

[13] E. Bouchaud, J. P. Bouchaud, D. S. Fisher, S. Ramanathan, and J. R. Rice, J. Mech. Phys. Solids 50, 1703 (2002).

[14] D. Bonamy, S. Santucci, and L. Ponson, Phys. Rev. Lett. 101, 045501 (pages 4) (2008).

[15] S. S. P. Parkin, M. Hayashi, and L. Thomas, Science 320, 190 (2008).

[16] H. Ohno, Nature Materials 9, 952 (2010).

[17] K.-J. Kim, J.-C. Lee, S.-M. Ahn, K.-S. Lee, C.-W. Lee, Y. J. Cho, S. Seo, K.-H. Shin, S.-B. Choe, and H.-W. Lee, Nature (London) 458, 740 (2009).

[18] D. Cule and T. Hwa, Phys. Rev. B 57, 8235 (1998).

[19] C. J. Bolech and A. Rosso, Phys. Rev. Lett. 93, 125701 (2004).

[20] S. Bustingorry, A. B. Kolton, and T. Giamarchi, Phys. Rev. B 82, 094202 (2010).

[21] A.-L. Barabási and H. E. Stanley, Fractal Concepts in Surface Growth (Cambridge, 1995), cambridge university press ed.

[22] S. Bustingorry, A. B. Kolton, and T. Giamarchi, Phys. Rev. B 85, 214416 (2012).

[23] A. A. Fedorenko, P. Le Doussal, and K. J. Wiese, Phys.
Rev. E 74, 041110 (2006).

[24] S. Bustingorry and A. B. Kolton, Papers in Physics 2, 1 (2009).

[25] P. Le Doussal and K. J. Wiese, Phys. Rev. E 79, 051105 (2009).

[26] A. A. Middleton and D. S. Fisher, Phys. Rev. B 47, 3530 (1993).

[27] O. Narayan and D. S. Fisher, Phys. Rev. B 46, 11520 (1992).

[28] A. B. Kolton, A. Rosso, T. Giamarchi, and W. Krauth, Phys. Rev. Lett. 97, 057001 (2006).

[29] A. B. Kolton, A. Rosso, T. Giamarchi, and W. Krauth, Phys. Rev. B 79, 184207 (2009).

[30] E. E. Ferrero, S. Bustingorry, A. B. Kolton, and A. Rosso, Comptes Rendus Physique (2013).

[31] A. Rosso and W. Krauth, Phys. Rev. E 65, 025101 (2002).

[32] A. Rosso and W. Krauth, Phys. Rev. Lett. 87, 187002 (2001).

[33] E. E. Ferrero, S. Bustingorry, and A. B. Kolton, Phys. Rev. E 87, 032122 (2013).

[34] P. Le Doussal, K. J. Wiese, and P. Chauve, Phys. Rev. B 66, 174201 (2002).

[35] J. Galambos, The asymptotic theory of extreme order statistics (Krieger, Malabar, 1987).

[36] A. Rosso, P. Le Doussal, and K. J. Wiese, Phys. Rev. B 75, 220201(R) (2007).

[37] A. B. Kolton, A. Rosso, E. V. Albano, and T. Giamarchi, Phys. Rev. B 74, 140201 (2006).

[38] Note that $\zeta^{\mathrm{RP}} \equiv \zeta_{\mathrm{L}}=(4-d) / 2$ in $d=1$, where $\zeta_{\mathrm{L}}$ is the Larkin exponent. This reflects the fact that the RP fixed point with $\zeta=0$ is unstable due to a self-generated random-force [34]. 